\title{
Fraturas Atípicas do Fémur Associadas a Terapêutica Prolongada com Bisfosfonatos
}

\author{
Atypical Femoral Fractures Associated with Long-Term Treatment with \\ Bisphosphonates
}

Francisco FLORES SANTOS ${ }^{1}$, José PINHEIRO DA SILVA², Paulo FELICÍSSIMO ${ }^{1}$

Acta Med Port 2013 Nov-Dec;26(6):746-750

RESUMO

Introdução: A terapêutica prolongada com bisfosfonatos tem sido associada a fraturas atípicas do fémur cujas características estão neste momento definidas.

Casos Clínicos: Apresentam-se dois casos clínicos de doentes do género feminino sob terapêutica com bisfosfonatos há mais de 10 anos e que foram admitidas na nossa instituição por fraturas dos fémures resultantes de traumatismos de baixa energia. Estas fraturas localizavam-se na região subtrocantérica e mediodiafisária do fémur apresentando, respectivamente, traço simples transversal e oblíquo curto, com espessamento da cortical externa. Ambas foram submetidas a tratamento cirúrgico com bom resultado clínico e radiológico.

Discussão: Os casos apresentados cumprem os critérios aceites para o diagnóstico de fratura atípica do fémur, ilustrando um efeito adverso grave da terapêutica prolongada com bisfosfonatos. A evidência científica ainda não estabeleceu esta associação de forma inequívoca. Por outro lado, a eficácia destes fármacos na prevenção de fraturas osteoporóticas está comprovada.

Conclusão: A fratura atípica do fémur pode constituir um efeito adverso grave da terapêutica prolongada com bisfosfonatos. A evidência científica continua a suportar a sua utilização, mas o clínico deverá estar alerta e acompanhar atentamente estes doentes.

Palavras-chave: Difosfonatos/ efeitos adversos; Fracturas do Fémur/ etiologia.

ABSTRACT

Introduction: Long-term treatment with bisphosphonates has been associated to atypical femoral fractures whose features are now clearly defined.

Clinical Cases: We present two cases of female patients under bisphosphonate treatment for over 10 years who were admitted to our institution for subtrochanteric and femoral shaft fractures after low-energy trauma. They presented, respectively, a transverse and a short oblique femoral fracture, with thickening of the lateral cortex. They underwent surgical treatment obtaining good functional and imaging result.

Discussion: These cases fulfill the established criteria for atypical femoral fracture, thereby illustrating a serious adverse event of long-term treatment with bisphosphonates. Such relationship has still not been clearly established by scientific evidence. However, its effectiveness in preventing osteoporotic fractures is well proven.

Conclusion: The atypical femoral fractures are possibly a serious adverse effect of the long-term treatment with bisphosphonates. Scientific evidence still supports its use, however, the physician must be aware of these events and closely follow-up these patients.

Keywords: Diphosphonates/ adverse effects; Femoral Fractures/ etiology.

\section{INTRODUÇÃO}

Os bisfosfonatos constituem a classe mais importante de agentes anti-reabsortivos utilizados para tratar doenças caracterizadas por reabsorção óssea mediada por osteoclastos como osteoporose, doença de Paget, doença óssea neoplásica. ${ }^{1}$ São fármacos muito eficazes na redução do risco de fraturas, incluindo as fraturas do colo do fémur e da região trocantérica. ${ }^{2}$

Os bisfosfonatos utilizados atualmente são compostos nitrogenados que atuam ligando-se ao osso onde vão atuar sobre os osteoclastos, inibindo a sua atividade e induzindo a apoptose. ${ }^{3}$ Como consequência existe também uma inibição da remodelação, tornando o osso adinâmico do ponto de vista celular o que permite a acumulação de lesões microscópicas que com o tempo podem culminar em fraturas de fadiga ${ }^{4}$. Com efeito, tem sido reportada a ocorrência de fraturas atípicas da região subtrocantérica e diafisária do fémur aparentemente relacionadas com a terapêutica pro- longada com bisfosfonatos. ${ }^{2}$

\section{CASO CLÍNICO UM}

Doente do género feminino, raça caucasiana, com 63 anos, com história pessoal de menopausa precoce aos 39 anos e tiroidectomia há oito anos pelo que foi medicada com levotiroxina. Aos 49 anos, na sequência de osteodensitometria compatível com osteoporose, iniciou terapêutica com cálcio 1200 mg/dia, vitamina D 250 Ul/dia e alendronato $10 \mathrm{mg} / \mathrm{dia}$, este último substituído por dose de $70 \mathrm{mg}$ semanal sete anos antes da fratura. No total, realizou terapêutica de forma continua ao longo de 14 anos.

Ao realizar atividades no domicílio sofreu uma queda em ortostatismo de uma altura de cerca de $50 \mathrm{~cm}$, associada a um movimento de rotação e sem trauma direto no solo, referindo dor súbita na coxa esquerda.

Foi admitida na nossa instituição, onde realizou uma

1. Serviço de Ortopedia. Hospital Fernando Fonseca. Amadora. Portugal.

2. Serviço de Ortopedia. Hospital Força Aérea. Lisboa. Portugal.

Recebido: 16 de Julho de 2013 - Aceite: 10 de Setembro de 2013 | Copyright @ Ordem dos Médicos 2013 


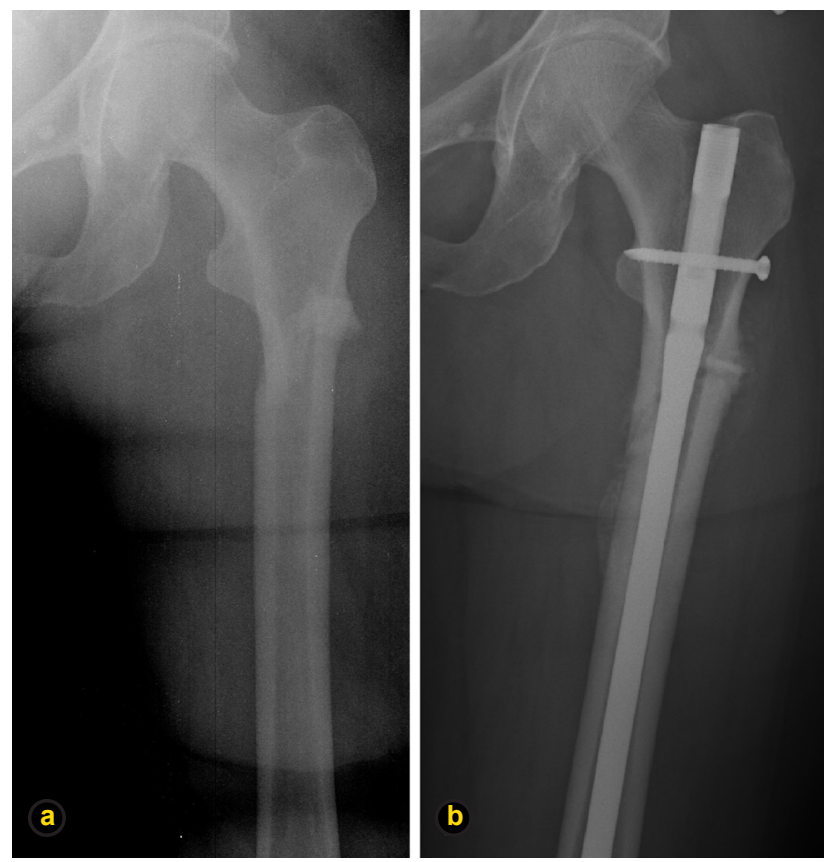

Figura 1 - Radiografias relativas ao caso clínico 1: a) fractura subtrocantérica do fémur esquerdo, de traço simples, oblíquo curto e espessamento da cortical externa a esse nível; b) pós-operatório de osteossíntese endomedular anterógrada. radiografia simples que revelou fratura subtrocantérica de traço simples, oblíquo curto com spike interno e aparente espessamento da cortical externa a este nível. (Fig. 1a). Foi tratada cirurgicamente por redução incruenta e osteossíntese endomedular com cavilha anterógrada (Fig. 1b). Fo imediatamente suspensa a terapêutica com bisfosfonatos, mantendo apenas a suplementação com cálcio e vitamina $\mathrm{D}$ nas doses descritas.

\section{CASO CLÍNICO DOIS}

Doente do género feminino, raça caucasiana, 72 anos, com diagnóstico de osteoporose, foi medicada com alendronato $10 \mathrm{mg} /$ dia que cumpriu durante cinco anos após os quais foi substituído pela associação de alendronato com vitamina D $70 \mathrm{mg} / 2800 \mathrm{UI}$ semanais durante os cinco anos seguintes.

Nos três meses que antecederam a fratura, referia coxalgia direita, pela qual foi avaliada e realizou radiografia e RMN que revelaram fratura de stress da cortical externa do fémur (Fig. 2). Foi-Ihe recomendada descarga e seguimento em consulta de ortopedia.

Na sequência de queda da própria altura, sofreu fratura diafisária do fémur direito, pelo que recorreu ao nosso hospital onde foi submetida a osteossíntese endomedular com cavilha anterógrada. O período de reabilitação decorreu do
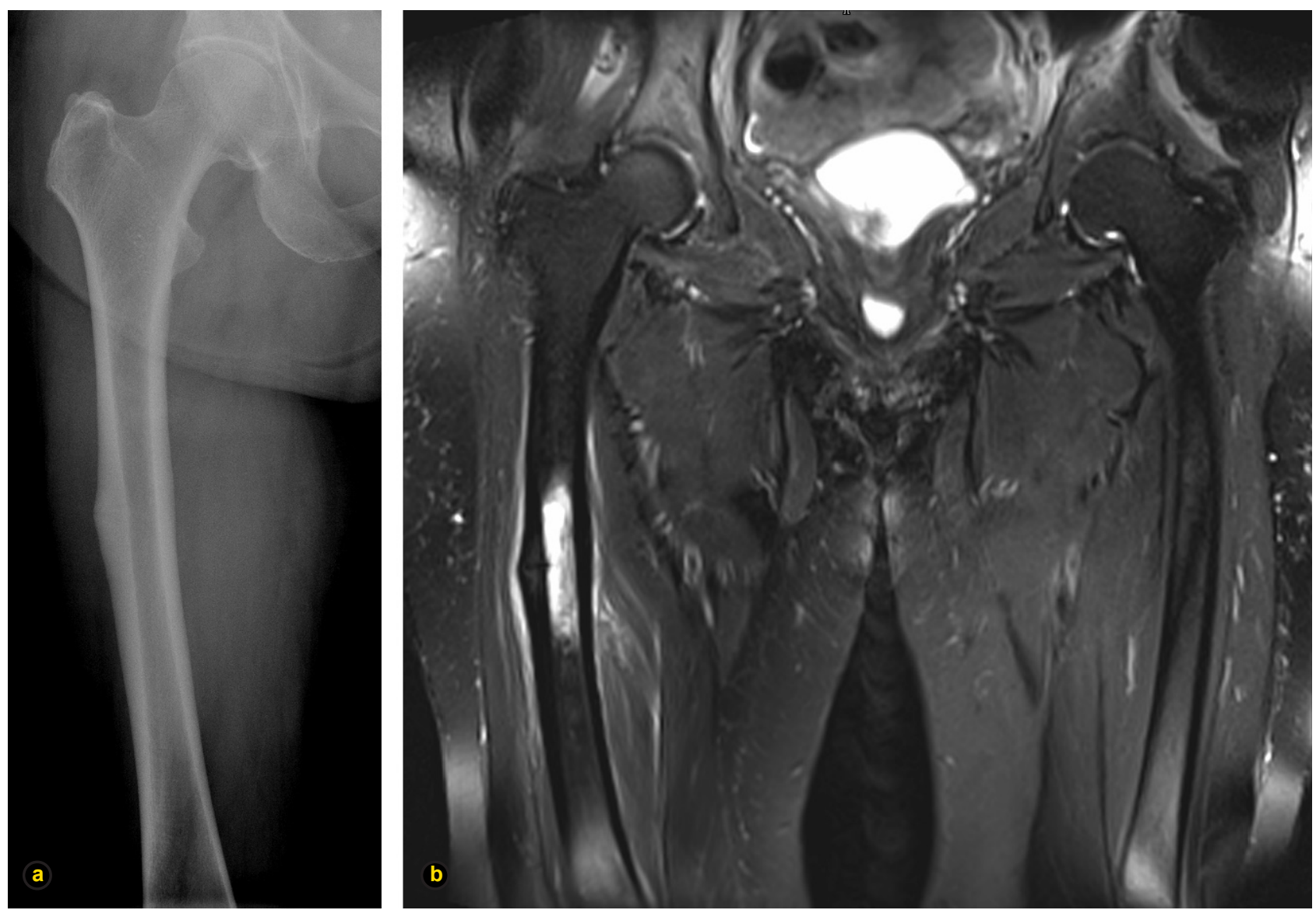

Figura 2 - Exames de imagem relativos à fratura do fémur direito do caso 2: a) Radiografia que revela traço de fratura da região diafisária do fémur com espessamento da cortical externa; b) ressonância magnética em T2 com evidente traço de fratura e edema ósseo da diáfise do fémur; 

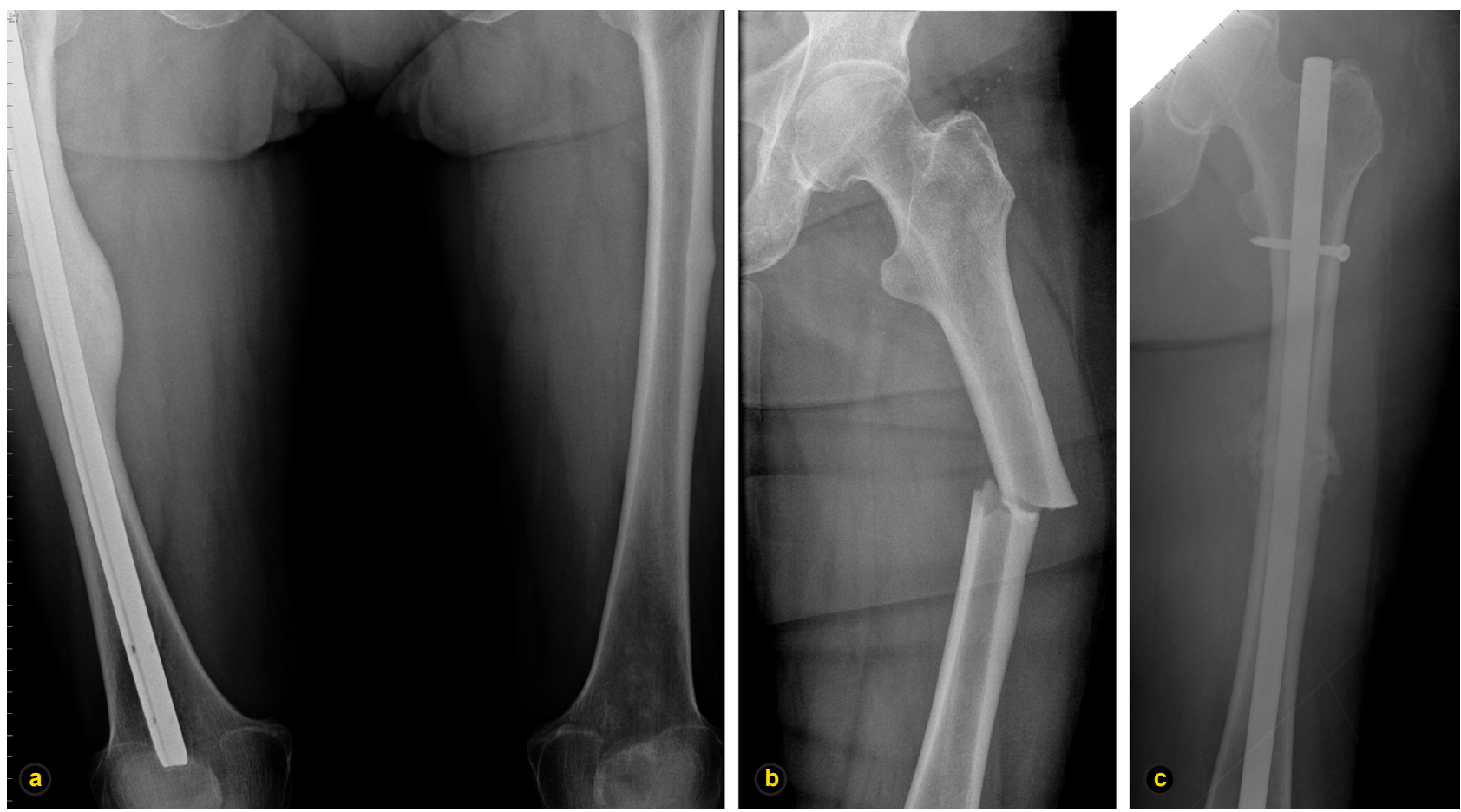

Figura 3 - Radiografias relativas à segunda fratura da doente do caso 2: a) diáfises dos fémures revelando pós-operatório de osteossíntese endomedular anterógrada à direita e imagem de espessamento da cortical externa esquerda; b) fratura de traço simples e transversal da região diafisária do fémur esquerdo; c) pós-operatório de osteossíntese endomedular anterógrada do fémur esquerdo.

modo usual, com recuperação funcional total, tendo retomado terapêutica com alendronato.

Dois anos após este episódio iniciou queixas semelhantes às anteriormente descritas no membro inferior contralateral. Realizou radiografia, que revelava espessamento da cortical externa da diáfise do fémur esquerdo (Fig. 3a). A osteodensitometria na extremidade proximal do fémur revelou um T-score de $-1,7$. Foi suspensa a terapêutica com alendronato e recomendada descarga, mas em nova queda da própria altura sofreu fratura à esquerda descrevendo dor antes do contacto com o solo (Fig. 3b). Foi novamente submetida a cirurgia com a mesma técnica e com resultado funcional sobreponível. A radiografia de pós-operatório às sete semanas evidenciou formação de calo ósseo (Fig. 3c). Neste momento a doente encontra-se assintomática sob terapêutica com cálcio $600 \mathrm{mg} /$ dia e vitamina D $400 \mathrm{UI} / \mathrm{dia}$.

\section{DISCUSSÃO}

Os bisfosfonatos são fármacos utilizados frequentemente na prática clínica e apresentam eficácia suportada pela evidência científica na prevenção de fraturas osteoporóticas. ${ }^{5}$

Recentemente foi reportada a ocorrência de fraturas atípicas do fémur que acrescem aos efeitos adversos previamente descritos. Essas fraturas são raras, em particular considerando os milhões de doentes que realizaram terapêutica com bisfosfonatos e comparando com a elevada frequência das fraturas proximais do fémur convencionais. ${ }^{2}$

Os resultados dos estudos nesta área têm sido divergentes quanto ao aumento de risco de fraturas atípicas não se tendo verificado um aumento significativo no risco de fraturas subtrocantéricas ou diafisárias do fémur num estudo de revisão de três grandes ensaios clínicos de Black et al. ${ }^{6}$ No entanto, um estudo caso-controlo por Park-Wyllie et al, obteve um aumento do risco desses eventos em muIheres pós-menopausicas a cumprir terapêutica há mais de cinco anos. ${ }^{7}$

Por esta razão, a American Society for Bone and Mineral Research criou um grupo de trabalho com o objetivo de abordar algumas questões como: revisão da informação existente, desenvolvimento de meios diagnósticos, linhas de investigação e recomendações para a prática clínica. ${ }^{2}$ Verificou-se que estas fraturas podem ocorrer em doentes que não realizam terapêutica com bisfosfonatos e a sua verdadeira incidência em indivíduos tratados e não tratados é ainda desconhecida. ${ }^{2}$ Um dos aspetos considerados importantes foi a definição de caso de fratura atípica cujas características específicas se encontram sumarizadas na Tabela $1 .^{2}$

Apresentamos dois casos de fraturas do fémur que satisfazem os critérios de fratura atípica (Tabela 1) e que ocorreram na sequência de traumatismos de baixa energia, em mulheres sob terapêutica com bisfosfonatos por período prolongado. As doentes não apresentavam outros antecedentes conhecidos, nem cumpriam medicação para além da descrita anteriormente.

No caso 1 e na segunda fratura do caso 2 , as doentes referem claramente a dor súbita na coxa antes da queda propriamente dita. No caso 2, documentou-se uma alteração da cortical femoral contralateral que precedeu uma fratura a esse nível. Nesta doente, a osteodensitometria prévia à segunda fratura não apresentava valores compatíveis 
Tabela 1 - Fratura atípica do fémur ${ }^{a}$

\section{Critérios major ${ }^{\mathrm{b}}$}

- Em qualquer localização na região subtrocantérica/diafisária do fémur

- Sem história de trauma ou trauma minor (queda da própria altura ou menos)

- Fratura de traço transverso ou oblíquo curto

- Não cominutiva

- Fraturas completas envolvem as duas corticais e apresentam um spike interno, as incompletas envolvem apenas o córtex externo

\section{Critérios minor ${ }^{\mathrm{b}}$}

- Reação periostal que envolve o córtex externo

- Aumento generalizado na espessura cortical da diáfise

- Sintomas prodrómicos - dor na região inguinal ou coxa

- Sintomas e fraturas bilaterais

- Atraso de consolidação

- Comorbilidade (ex: deficiência de vitamina D, Artrite reumatóide, hipofosfatasia)

- Utilização de fármacos (bisfosfonatos, glucocorticoides, inibidores da bomba de protões)

a) Estão excluídas as fraturas do colo do fémur, da região intertrocantérica com extensão espiralada à região subtrocantérica, fraturas patológicas associadas a lesão neoplásica primária ou metastática e fraturas periprotésicas.

b) Todos os critérios major têm que estar presentes. Não é necessário verificar-se qualquer dos critérios minor

com osteoporose (T-score $<-2,5)$.

Os casos apresentados ilustram a ocorrência de fraturas atípicas do fémur, para as quais não foi determinada uma causa evidente. No caso 1 coexiste patologia tiroideia medicada com levotiroxina, que segundo alguns estudos, pode associar-se a diminuição da densidade da massa óssea e desta forma aumentar o risco de fraturas. ${ }^{8}$ Ao contrário dos casos apresentados, os associados à terapêutica com levotiroxina não obedecem às características apresentadas na tabela 1 , entre as quais a localização subtrocantérica ou diafisária. ${ }^{8} \mathrm{O}$ denominador comum entre os nossos dois casos parece ser a terapêutica prolongada com bisfosfonatos, colocando-nos perante aquele que parece ser um efeito adverso grave da terapêutica com estes fármacos.

Este aspeto deve ser tido em consideração na prática clínica, tanto na prescrição do fármaco como na instituição de um intervalo terapêutico após administração prolongada. O clínico deve estar sensibilizado para este potencial efeito secundário, realizando uma radiografia do fémur no caso de o doente apresentar coxalgia. ${ }^{9}$

Outros relatos de caso têm sido publicados levantando esta questão, mas não existe evidência suficiente que suporte a hipótese de a terapêutica com bisfosfonatos, por si só, levar à ocorrência destas fraturas atípicas associadas a trauma de baixa energia. Uma das limitações de vários estudos é de avaliarem a ocorrência de fraturas baseada apenas na localização, ignorando o facto de estas não poderem ser consideradas sempre como atípicas mas, em muitos casos, como parte da história natural da osteoporose. Um aspeto adicional a ser considerado é a patologia concomitante e a utilização de outros fármacos, como corticosteroides, que podem constituir fatores de risco para a ocorrência de fraturas subtrocantéricas do fémur.

Provavelmente, apenas ensaios clínicos randomizados e controlados poderão avaliar correctamente esta relação causal. Até à data não o comprovaram mas tratavam-se de ensaios clínicos que não tinham em atenção este problema em particular aquando da sua execução, tendo sido realizada apenas uma análise retrospetiva dos dados.

A importância e eficácia dos bisfosfonatos na prevenção das fracturas osteoporóticas comuns é reconhecida e a sua associação com fraturas atípicas é ainda controversa, pelo que a relação risco-benefício continua a favorecer a sua utilização. ${ }^{9}$

\section{CONCLUSÃO}

Inúmeros relatos de caso colocam a questão da segurança da terapêutica prolongada com bisfosfonatos em doentes osteoporóticos e oncológicos. O clínico deve estar sensibilizado para este potencial efeito adverso. Dada a sua comprovada ação na prevenção das fraturas osteoporóticas, estes fármacos deverão continuar a ser utilizados mas com precaução e seguimento médico regular, equacionando estabelecer um período de pausa em casos de terapêutica prolongada.

Torna-se urgente obter mais informação científica que permita estabelecer doses, períodos máximos de tratamento e eventual interrupção periódica para a utilização segura destes fármacos.

\section{CONFLITO DE INTERESSES}

Os autores declaram que não houve conflito de interesses na realização deste trabalho.

\section{FONTES DE FINANCIAMENTO}

Os autores declaram a inexistência de fontes de financiamento externas. 


\section{REFERÊNCIAS}

1. Morris CD, Einhorn TA. Bisphosphonates in orthopaedic surgery. J Bone Joint Surg Am. 2005;87:1609-18.

2. Shane E, Burr D, Ebeling PR, Abrahamsen B, Adler RA, Brown TD, et al. Atypical subtrochanteric and diaphyseal femoral fractures: report of a task force of the American Society for Bone and Mineral Research. J Bone Miner Res. 2010;25:2267-94.

3. Gehrig J, Lane J, O'Connor MI. Osteoporosis: Management and treatment strategies for orthopaedic surgeons. J Bone Joint Surg Am. 2008;90:1362-74.

4. Schilcher J, Michaelsson K, Aspenberg P. Bisphosphonate use and atypical fractures of the femoral shaft. N Engl J Med. 2011;364:1728-37.

5. Favus MJ. Bisphosphonates for Osteoporosis. N Engl J Med. 2010;363:2027-35.
6. Black DM, Kelly MP, Genant HK, Palermo L, Eastell R, Bucci-Rechtweg C. Bisphosphonates and fractures of the subtrochanteric or diaphyseal femur. N Engl J Med. 2010;362:1761-71.

7. Park-Wyllie LY, Mamdani MM, Juurlink DN, Hawker GA, Gunraj N, Austin PC, et al. Bisphosphonate use and the risk of subtrochanteric or femoral shaft fractures in older women. JAMA. 2011;305:783-9.

8. Leese, GP, Flynn R. V. Levothyroxine dose and fractures in older adults. BMJ. 2011;342:d2238

9. Rizzoli R, Akesson K, Bouxsein M, Kanis JA, Napoli N, Papapoulos S, et al. Subtrochanteric fractures after long-term treatment with bisphosphonates: a European Society on Clinical and Economic Aspects of Osteoporosis and Osteoarthritis, and International Osteoporosis Foundation Working Group Report. Osteoporos Int. 2011;22:373-90.

\section{was}

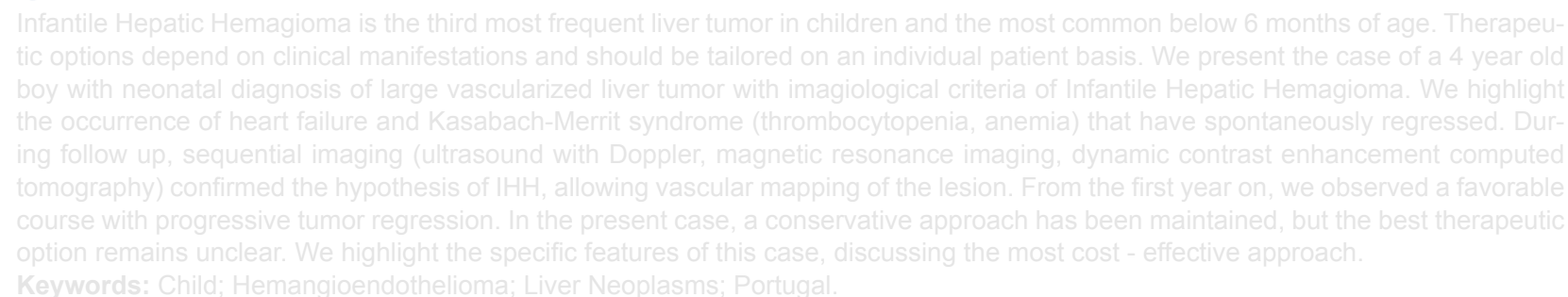




\section{Fraturas Atípicas do Fémur Associadas a Terapêutica Prolongada com Bisfosfonatos \\ Acta Med Port 2013:26:746-750}

Publicado pela Acta Médica Portuguesa, a Revista Científica da Ordem dos Médicos

Av. Almirante Gago Coutinho, 151

1749-084 Lisboa, Portugal.

Tel: +351218428 215

E-mail: submissao@actamedicaportuguesa.com

www.actamedicaportuguesa.com

ISSN:0870-399X | e-ISSN: 1646-0758

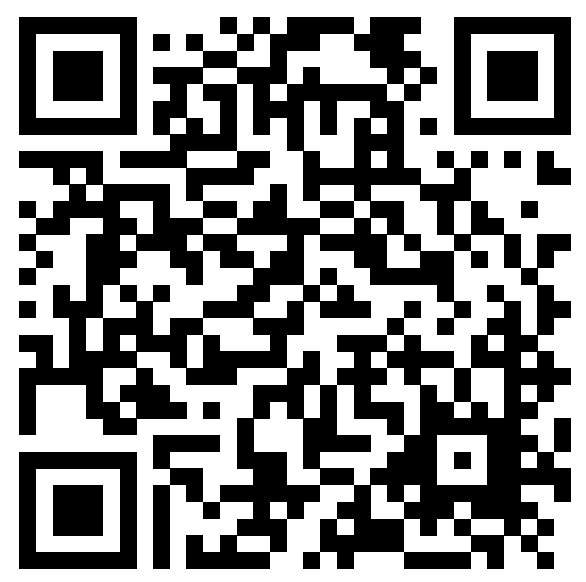

